\title{
Cosmic Mach Number: A Sensitive Probe for the Growth of Structure
}

\author{
Yin-Zhe Ma, ${ }^{a}$ Jeremiah P. Ostriker ${ }^{b, a}$ Gong-Bo Zhao ${ }^{c, d}$ \\ ${ }^{a}$ Kavli Institute for Cosmology and Institute of Astronomy, University of Cambridge, Madingley \\ Road, Cambridge, CB3 0HA, UK \\ ${ }^{b}$ Department of Astrophysical Sciences, Princeton University, 4 Ivy Lane, Princeton, NJ, U.S.A \\ ${ }^{c}$ Institute of Cosmology and Gravitation, University of Portsmouth, Dennis Sciama Building, Portsmouth, \\ PO1 3FX, UK \\ ${ }^{d}$ National Astronomy Observatories, Chinese Academy of Science, A20 Datun Road, Chaoyang \\ District, Beijing, China \\ E-mail: mayinzhe@phas.ubc.ca, ostriker@princeton.edu, Gong-Bo.Zhao@port.ac.uk
}

\begin{abstract}
We investigate the potential power of the Cosmic Mach Number (CMN), which is the ratio between the mean velocity and the velocity dispersion of galaxies as a function of cosmic scales, to constrain cosmologies. We first measure the CMN from 4 catalogs of galaxy peculiar velocity surveys at low redshift $(z \in[0.002,0.03])$, and use them to contrast cosmological models. Overall, current data is consistent with the WMAP7 $\Lambda$ CDM model. We find that the CMN is highly sensitive to the growth of structure on scales $k \in[0.01,0.1] \mathrm{h} / \mathrm{Mpc}$ in Fourier space. Therefore, modified gravity models, and models with massive neutrinos, in which the structure growth generically deviates from that of the $\Lambda \mathrm{CDM}$ model in a scale-dependent way, can be well differentiated from the $\Lambda \mathrm{CDM}$ model by using future $\mathrm{CMN}$ data.
\end{abstract}

Keywords: Cosmology: large scale structure, observations, theory; Galaxy: kinematics and dynamics

ArXiv ePrint: 1106.3327 


\section{Contents}

1 Introduction 1

2 Statistics of Cosmic Mach Number 2

3 A sensitive test of growth of structure 5

$3.1 \quad f(R)$ gravity model $\quad 5$

3.2 Massive neutrinos $\quad 5$

$\begin{array}{lll}3.3 & \text { Fisher Matrix forecast } & 6\end{array}$

\section{Conclusion}

\section{Introduction}

The Cosmic Mach Number (hereafter CMN) can provide a robust measure of the shape and growth rate of the peculiar velocity power spectrum of the galaxies in the universe. One considers a region of a given size $r$ in the universe, and compares the bulk motion of the sphere with the random velocities within that region. The bulk motion provides a measurement of the forces on the region from irregularities external to it, so it measures the amplitude of perturbations on scales much larger than the region, whereas the random motions within the comoving region reflect gravitational perturbations on scales smaller than $r$. Thus their ratio, $M(r)$, depends on the shape of the perturbation spectrum while independent of its amplitude.

The concept was introduced by Ostriker et al. [11] as a cosmological metric that would be relatively independent of the "bias" of the test particles being observed and also relatively independent of the then quite uncertain perturbation amplitude. They concluded that although the existing data were poor, they gave estimates of the CMN that appeared to be inconsistent with the then popular CDM model with $\Omega_{m}=1$ but seemed to prefer the open universe model instead. In a certain sense, the application of this test, correctly predicted the currently best validated cosmological models with a value of $\Omega_{m}$ in the range of $0.2-0.3$.

Subsequent to the original paper, Strauss et al. [14] found again that the CDM models with $\Omega_{m}=1$ remained inconsistent with the better data they used, but some non-standard models passed the test (see also Suto et al. $[15,16])$. Nagamine et al. [10] looked at $\Lambda$ CDM models and found better agreement, but the then $\Lambda \mathrm{CDM}$ model with $\Omega_{m}=0.37$ again produced too high values of $M$ over the range of $3-40 \mathrm{Mpc} / \mathrm{h}$, whereas a model with $\Omega_{m} \sim 0.2$ (actually closer to WMAP 7-yr constraint (Komatsu et al. [9])) was more consistent with the observations. In addition, there are various other papers discussing the issues related to the $\mathrm{CMN}$, such as non-linear clustering properties of dark matter on the CMN measurement (Gramann et al. [6]), and constraints on the CMN from SunyaevZeldovich effect (Atrio-Barandela et al. [2]).

This history leads us to re-examine the issue in light of the much better knowledge now available from both the new peculiar velocity data and the range of models remaining plausible given the current cosmological constraints. In this paper, we will develop a new statistical tool to measure the $\mathrm{CMN}$ from the peculiar velocity surveys, and investigate the power of the $\mathrm{CMN}$ to distinguish various cosmological models, especially in the aspects of differentiating the $\Lambda \mathrm{CDM}$ model from variance with non-trivial growth function provided by Modified Gravity (hereafter MG) models, and from models with massive neutrinos. 

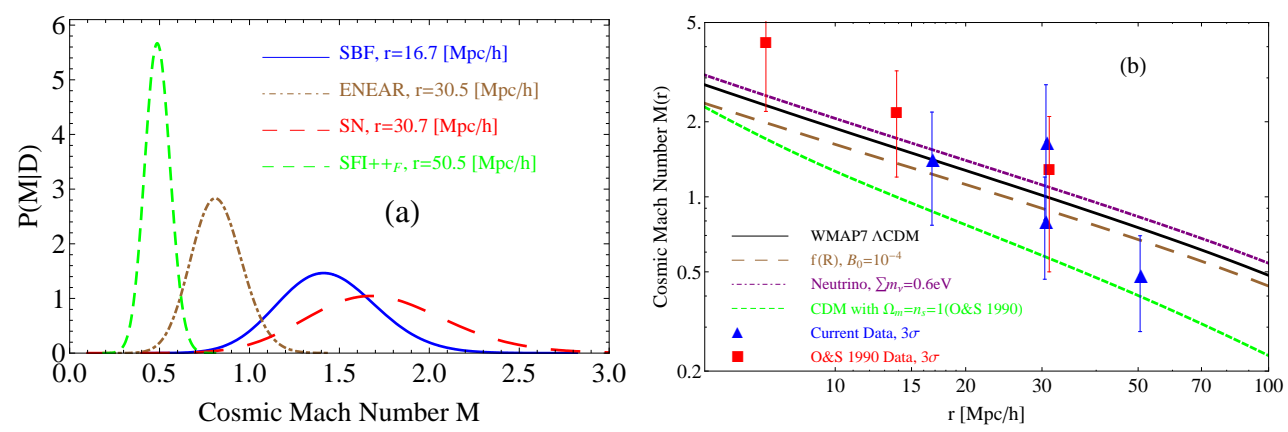

Figure 1. (a): The 1-D posterior distribution of the CMN $M(r)$ from 4 different catalogs. (b): The comparison between the CMN data ( $3 \sigma$ CL.) and theoretic prediction: blue data points are the CMN data from posterior distributions shown in panel (a); red data points are the data used in Ostriker et al. [11]. The black line is the CMN prediction from WMAP 7-yr best-fit values (Komatsu et al. [9]); the green dashed line is calculated by using (1990) 'popular' CDM cosmological parameters $n_{s}=\Omega_{m}=1$ and $h=0.5$ (Ostriker et al. [11]); the brown dashed and purple dot-dashed lines are the CMN from $f(R)$ model with $B_{0}=10^{-4}$ and from $\Lambda$ CDM model with neutrino mass $\sum m_{\nu}=0.6 \mathrm{eV}$.

\section{Statistics of Cosmic Mach Number}

In linear perturbation theory, the power spectrum of the velocity divergence $(\theta \equiv \nabla \cdot \mathbf{v})$ is related to the power spectrum of density fluctuations via $P_{\theta \theta}(k, z)=f^{2}(k, z) P(k, z)$, where $f(k, z) \equiv$ $-d \ln \delta / d \ln (1+z)$, and $\delta$ is the density perturbation of matter. Since the data in our application are at very low-redshift, we assume that they have the same redshift $z=0$ throughout and drop the $z$ dependence for brevity. The mean square velocity dispersion $\left\langle\sigma^{2}(r)\right\rangle$ and mean square bulk flow $\left\langle V^{2}(r)\right\rangle$ in a window of size $r$ can be calculated as Nagamine et al. [10], Ostriker et al. [11]

$$
\begin{aligned}
\left\langle V^{2}(r)\right\rangle & =\frac{H_{0}^{2}}{2 \pi^{2}} \int_{0}^{\infty} d k P_{\theta \theta}(k) W(k r), \\
\left\langle\sigma^{2}(r)\right\rangle & =\frac{H_{0}^{2}}{2 \pi^{2}} \int_{0}^{\infty} d k P_{\theta \theta}(k)[1-W(k r)],
\end{aligned}
$$

where $W(x)=\left[3(\sin x-x \cos x) / x^{3}\right]^{2}$ is a top-hat window function. Note that $W(x) \sim 1(x \lesssim 1)$ and $W$ drops to 0 quickly when $x>1$.

Thus $W$ effectively changes the integral limits of the above formula as

$$
\begin{aligned}
\left\langle V^{2}(r)\right\rangle & \simeq \frac{H_{0}^{2}}{2 \pi^{2}} \int_{0}^{1 / r} d k P_{\theta \theta}(k), \\
\left\langle\sigma^{2}(r)\right\rangle & \simeq \frac{H_{0}^{2}}{2 \pi^{2}} \int_{1 / r}^{\infty} d k P_{\theta \theta}(k)=C-\left\langle V^{2}(r)\right\rangle .
\end{aligned}
$$

where $C=\frac{H_{0}^{2}}{2 \pi^{2}} \int_{0}^{\infty} d k P_{\theta \theta}(k)$ is a normalization constant. We can see that the velocity dispersion $\left\langle\sigma^{2}(r)\right\rangle$ can be well approximated as a flipped signed large scale bulk flow $\left\langle V^{2}(r)\right\rangle$ with an offset $C$. This is an important feature since it guarantees that no calculation for the nonlinearities is needed to evaluate the velocity dispersion.

The CMN on different scales of the patches is defined as

$$
M(r) \equiv \sqrt{\frac{\left\langle V^{2}(r)\right\rangle}{\left\langle\sigma^{2}(r)\right\rangle}}=\left[\frac{C}{C-\left\langle V^{2}(r)\right\rangle}-1\right]^{1 / 2} .
$$


Thus it basically measures the shape of $P_{\theta \theta}$ by contrasting $\int d k P_{\theta \theta}(k)$ on large, and small scales. Using Eqs (2.1) and (2.3), one can reconstruct $P_{\theta \theta}(k)$ up to an overall constant $A$,

$$
P_{\theta \theta}(k=1 / r)=A \frac{M(r) r^{2}}{\left[1+M^{2}(r)\right]^{2}} \frac{d M(r)}{d r} .
$$

Directly measuring $P_{\theta \theta}$ from peculiar velocity surveys is challenging, but the measurement of CMN is much easier, as we show later. Fortunately, CMN has the same information as $P_{\theta \theta}$, except for the overall amplitude, which can be easily determined by probes such as the Cosmic Microwave Background (CMB).

For a peculiar velocity catalog with $N$ objects, the CMN $M$ can be written as $M=|\mathbf{u}| / \sigma_{*}$, where $\mathbf{u}$ denotes the bulk flow velocity, which is a streaming motion of galaxies towards some particular direction, and $\sigma_{*}$ stands for the small scale velocity dispersion. Unfortunately, neither $\mathbf{u}$ nor $\sigma_{*}$ is a direct observable. For each galaxy peculiar velocity catalog, what we observe is the line of sight velocity $S_{n}$ with measurement error $\sigma_{n}$ for the $n$th galaxy. Then one can construct a joint likelihood function for $\mathbf{u}$ and $M$ by contrasting $S_{n}$ with the line-of-sight projection of the bulk flow $\hat{r}_{n, i} u_{i}$. The uncertainty in $\left(S_{n}-\hat{r}_{n, i} u_{i}\right)$ is simply $\left(\sigma_{n}^{2}+\sigma_{*}^{2}\right)^{\frac{1}{2}}$, where $\sigma_{*}=|\mathbf{u}| / M$ is given by the definition of the CMN. Therefore, the likelihood function takes the form of,

$$
L(\mathbf{u}, M)=\prod_{n=1}^{N} \frac{1}{\left[\sigma_{n}^{2}+(|\mathbf{u}| / M)^{2}\right]^{\frac{1}{2}}} \times \exp \left(-\frac{\left(S_{n}-\hat{r}_{n, i} u_{i}\right)^{2}}{2\left(\sigma_{n}^{2}+(|\mathbf{u}| / M)^{2}\right)}\right) .
$$

One can then marginalize over the 3D bulk flow vector $\mathbf{u}$ to obtain the distribution of $M$ for each survey.

We use four different catalogs of galaxy peculiar velocity surveys, namely, SBF (Tonry et al. [18]), ENEAR (Bernardi et al. [3], da Costa et al. [4], Wenger et al. [20]), Type Ia Supernovae (SN) (Tonry et al. [19]), and SFI++ $F$ (Springob et al. [13]), to constrain the CMN by using Eq. (2.5). To calculate the characteristic depth $\bar{r}$ of each catalog, we use error-weighted depth as $\bar{r}=$ $\sum_{n} w_{n} r_{n} / \sum_{n} w_{n}$, where $w_{n}=1 /\left(\sigma_{n}^{2}+\sigma_{*}^{2}\right)$. We marginalize over the 'bulk flow' velocity $\mathbf{u}$ in the 4-D parameter space and obtain the 1-D posterior distribution of $M$ as shown in panel (a) of Fig. 1. In the panel (a), one can see that the distribution of the CMN is very Gaussian, and the width depends primarily on the number of data entries in each catalog. In addition, since each catalog probes the CMN on various depths, different catalogs form a complimentary set of tests of cosmic structures.

In panel (b) of Fig. 1, we put together the old (1990) and current CMN data with various predictions computed from theoretic models: The [Blue] triangle data points are the current CMN values and variances computed from likelihood (2.5) by using the four-catalogs, which provide more and deeper data than those used by Ostriker et al. [11] ([Red] square data).

The $\Lambda$ CDM model with WMAP 7-yr best-fit parameters $\left(\Omega_{m}=0.271, h=0.704, n_{s}=\right.$ $0.967, \Omega_{b}=0.0455$, [Black] solid line) is mildly consistent with the current CMN data at $3 \sigma \mathrm{CL}$. In comparison, we overplot the theoretical $M(r)$ ([Green] dashed line) by using 1990's 'popular' CDM parameters. One can immediately understand the reason why Refs. Ostriker et al. [11], Strauss et al. [14] claimed that there was a strong conflict between the data ([Red] points) and popular CDM model ([Green] dashed line). There was in fact no inconsistency between data and a model with $\Omega \approx 0.25$ and $n_{s}=1$. However, with the up-to-date data and strongly constrained $\Lambda$ CDM cosmology, one can see that the CMN data is consistent with the $\Lambda \mathrm{CDM}$ prediction out to scale around $50 \mathrm{Mpc} / \mathrm{h}$. Note that the small scale CMN can be contaminated by the nonlinearity of the growth. For instance, using the fitting formulae of non-linear $P_{\theta \theta}$ proposed in Jennings et al. [7], we find that CMN is suppressed about $21 \%(18 \%)$ on scale of $15 \mathrm{Mpc} / \mathrm{h}(20 \mathrm{Mpc} / \mathrm{h})$. Thus nonlinearities must be considered when 

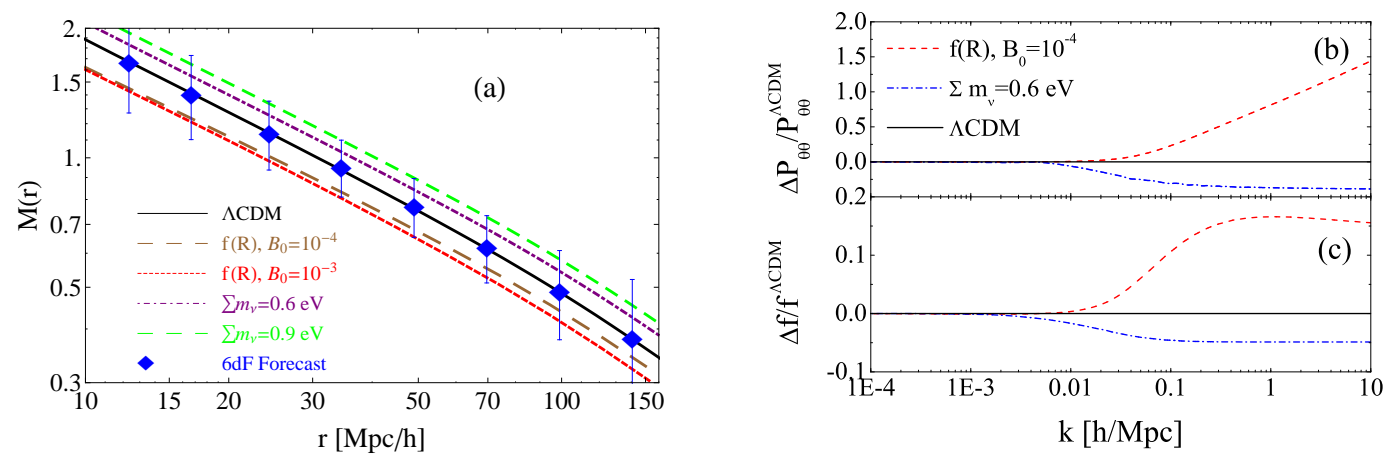

Figure 2. (a): Forecast $1 \sigma$ errors of the $\mathrm{CMN}$ from $6 \mathrm{dF}$ survey, in which we assume the data is around our local region of the universe. Beside $\Lambda \mathrm{CDM}$ model, we plot $B_{0}=10^{-4}$ ([Brown] dashed line) and $10^{-3}$ ([Red] dashed line) for the $f(R)$ model, and $\sum m_{\nu}=0.6 \mathrm{eV}$ ([Purple] dot-dashed line) and 0.3eV ([Green] dashed line). (b): $\Delta P_{\theta \theta} / P_{\theta \theta}$ for $f(R)$ (dashed line) and massive neutrino (dot-dashed line) models. (c): Same comparison as (b) but for growth function $\Delta f / f$.

using the CMN data. On the other hand, numerical simulation can be used to quantify the effect of non-linearities.

To investigate the prospective accuracy of the CMN achievable in future surveys, we perform a forecast for the on-going $6 \mathrm{dF}$ peculiar velocity survey (Jones et al. [8]). The redshift distribution of galaxies for the $6 \mathrm{dF}$ survey is $n_{g}(z)=A z^{\gamma} e^{-\left(z / z_{p}\right)^{\gamma}}$, where $z_{p} \simeq 0.0446, \gamma \simeq 1.6154$ and $A \simeq 622978$ (Jones et al. [8]). It peaks around $z \approx 0.05$ and extends till $z \approx 0.15$. We assume that the $6 \mathrm{dF}$ survey can measure the peculiar velocity for 12000 brightest early-type galaxies, which is roughly $10 \%$ of the total sample, and they are located at $z \lesssim 0.05$, corresponding to the depth $r \lesssim 150 \mathrm{Mpc} / \mathrm{h}$. We further assume that the measurement error for line-of-sight velocity is around $20 \%$, which is a typical error for the fundamental plane distance measurement. We divide these data into different redshift bins, and in each shell $(r, r+d r)$, we calculate the Fisher Matrix value for the CMN $F_{M M}$ from Eq.(2.5) which leads to the forecasted error of the CMN as

$$
\sigma_{M(r)} \simeq \frac{M(r)}{\sqrt{2 N(r)}}\left[1+\frac{\sigma_{n}^{2}}{(u(r) / M(r))^{2}}\right],
$$

where $N(r)$ is the number of data points in the shell $(r, r+d r)$, and $u(r)$ is the average bulk flow magnitude on depth $\mathrm{r}$. We plot these forecast data in the panel (a) of Fig. 2. Comparing with panel (b) of Fig. 1, We find the full range of the CMN data on scales $[10,150](\mathrm{Mpc} / \mathrm{h})$ from $6 \mathrm{dF}$ can improve the constraint on the variation of the scale-dependent growth factor significantly. We summarize the experimental conditions for future experiment that can sharpen the CMN test: (1) there should be considerably more galaxy samples $\left(\gtrsim 10^{4}\right)$ on scales $[10,150] \mathrm{Mpc} / \mathrm{h} ;(2)$ the smaller the measurement error $\sigma_{n}$ is, and the larger sky area it covers, the better it can reduce the overall error of $M$. One should also notice that, when the CMN data is used to constrain cosmology, the cosmic variance $(\mathrm{CV})$ due to the limited volume of the survey should be taken into account. The cosmic variance $\sigma_{\mathrm{CV}}(r)$ can be estimated as

$$
\left[\frac{\sigma_{\mathrm{CV}}(r)}{M(r)}\right]^{2}=\frac{1}{4}\left\{\left[\frac{\sigma_{A}(r)}{A(r)}\right]^{2}+\left[\frac{\sigma_{B}(r)}{B(r)}\right]^{2}\right\}
$$

where $A$ and $B$ stand for $\left\langle V^{2}(r)\right\rangle$ and $\left\langle\sigma^{2}(r)\right\rangle$ respectively, as defined in Eq (2.1), and $\sigma_{A}(r)$ and $\sigma_{B}(r)$ can be calculated as, 


$$
\begin{aligned}
& \sigma_{A}(r)=\frac{H_{0}^{2}}{2 \pi^{2} \sqrt{2 \pi}} \int \frac{1}{\sqrt{k^{2} \Delta k V}} P_{\theta \theta}(k) W(k r) d k, \\
& \sigma_{B}(r)=\frac{H_{0}^{2}}{2 \pi^{2} \sqrt{2 \pi}} \int \frac{1}{\sqrt{k^{2} \Delta k V}} P_{\theta \theta}(k)[1-W(k r)] d k .
\end{aligned}
$$

where $\Delta k$ and $V$ are the width of the $k$ bin and the volume of the survey respectively, and $W$ is the same window function as in Eq (2.1). We found that for the current velocity surveys of SBF, ENEAR, SN and SFI++ ${ }_{F}, \mathrm{CV}$ is $21,19,5$ and $10 \%$ of the measurement error. For the $6 \mathrm{dF}$ survey, $\mathrm{CV}$ is smaller than $5 \%$ of the statistical errors in all the bins.

\section{A sensitive test of growth of structure}

Since the CMN measures the shape of the peculiar velocity power spectrum $P_{\theta \theta}$ by design, it is sensitive to any distortion of $P_{\theta \theta}$. In the $\Lambda \mathrm{CDM}$ model, the growth is scale-independent. However, in the modified gravity models, and models with massive neutrinos, the growth is generically scaledependent, thus $P_{\theta \theta}$ for these models is a distorted version comparing with the $\Lambda \mathrm{CDM}$ model, making the $\mathrm{CMN}$ an excellent tool to distinguish these models from $\Lambda \mathrm{CDM}$. In the following of this section, we make separate discussion on the alternative theory of gravity and massive neutrino, and their prospective discrimination from CMN.

\section{1 $f(R)$ gravity model}

In a viable $f(R)$ model, where the Einstein-Hilbert action is extended to be a general function of the Ricci scalar $R$, the effective value of Newton's constant $G_{\text {eff }}$ has both time and scale dependence, namely, $G_{\text {eff }}=\mu(a, k) G$, where $\mu(a, k)=\left(1+\frac{4}{3} \lambda^{2} k^{2} a^{4}\right) /\left(1+\lambda^{2} k^{2} a^{4}\right)$, and $G$ is the Newton's constant in general relativity (GR) (Giannantonio et al. [5], Zhao et al. [21]). The only free parameter is $\lambda^{2}$, which quantifies the Compton wavelength of the scalar field $f_{R} \equiv d f / d R$ and characterizes the scale-dependent growth rate. It is more convenient to re-define a dimensionless $B_{0}$ which is $B_{0}=2 H_{0}^{2} \lambda^{2} / c^{2}$, and $B_{0}=0$ for GR (Giannantonio et al. [5]).

In panels (b) and (c) of Fig. 2, we show the relative difference in $P_{\theta \theta}$ and $f$ with respective to that in the $\Lambda \mathrm{CDM}$ model for a $f(R)$ model with $B_{0}=10^{-4}$ (dashed lines). The growth rate for $f(R)$ model is enhanced on scales of $k \gtrsim 0.01 \mathrm{~h} / \mathrm{Mpc}$, thus the integration of $P_{\theta \theta}(k)$ cumulates the deviation in $k$-space and exhibits the substantial difference of CMN between $\Lambda$ CDM and $f(R)$ model. Unfortunately, this substantial difference with $\Lambda$ CDM is not observable by the current CMN data due to the large errors, as shown in panel (b) of Fig. 1. However, as a result shown in panel (a) of Fig. 2, a $0.01 \%$ of $B_{0}$ in $f(R)$ model can produce a $20 \%$ suppression in the CMN, which is potentially observable by $6 \mathrm{dF}^{1}$.

\subsection{Massive neutrinos}

Similarly, the CMN is sensitive to the neutrino mass. When neutrinos became non-relativistic and the Universe was deeply in the matter dominated era, neutrino thermal velocities damped out the perturbation under the characteristic scale $k_{\mathrm{nr}} \simeq 0.018 \Omega_{m}^{1 / 2}\left(\sum m_{\nu} / 1 \mathrm{eV}\right) \mathrm{h} / \mathrm{Mpc}$, suppressing the power spectrum on small scales. On scales greater than $k_{\mathrm{nr}}$, neutrinos affect the overall expansion of the Universe and therefore shift the peak of power spectrum to larger scales. In panels (b) and (c) of

\footnotetext{
${ }^{1}$ We compare the forecast of $6 \mathrm{dF}$ constraint with the $B_{0}=10^{-4}, 10^{-3} \mathrm{f}(\mathrm{R})$ model in Fig. 2. This is because the constraints from CMB, Type Ia supernovae and ISW effect have already set $B_{0}<0.1$ [5]
} 

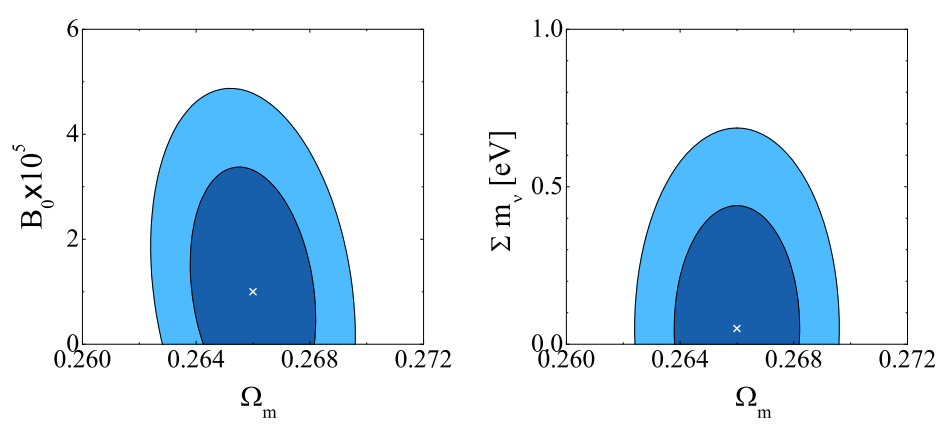

Figure 3. The 68 and $95 \%$ CL contour plots for $B_{0}$, neutrino mass and $\Omega_{m}$ derived from a combined dataset of WMAP, UNION2 and 6dF surveys using Fisher forecast. See the text for details.

Fig. 2, we can see that neutrino with mass of $0.6 \mathrm{eV}$ can suppress the power spectrum on scales of $k \gtrsim 0.01 \mathrm{~h} / \mathrm{Mpc}$ significantly, which exactly falls in the detection window of the CMN. Therefore, the cumulative 'integral' of $P_{\theta \theta}(k)$ in CMN can manifest the neutrino free-streaming effect by enhancing its value on all scales. Comparing with panel (b) of Fig. 1 and panel (a) of Fig. 2, we find that although the current data is weak in discriminating the massive neutrino, future CMN data is potentially able to distinguish the nonzero neutrino mass.

\subsection{Fisher Matrix forecast}

We are aware that the possible degeneracy among cosmological parameters might dilute the constraints, therefore we employ a Fisher matrix forecast (Tegmark et al. [17]) to study the power of $\mathrm{CMN}$ on cosmological parameter constraints. The Fisher matrix for $\mathrm{CMN}$ is,

$$
F_{\mu \nu}=\sum_{i, j} \frac{\partial M\left(r_{i}\right)}{\partial p_{\mu}} \operatorname{Cov}^{-1}\left[M\left(r_{i}\right), M\left(r_{j}\right)\right] \frac{\partial M\left(r_{j}\right)}{\partial p_{\nu}},
$$

where $p$ denotes the cosmological parameter, and Cov is the data covariance matrix for CMN. We include the cosmic variance of $\mathrm{CMN}$ in our analysis. We make a forecast for two models: $A=\left\{B_{0}, \mathrm{CP}\right\}, \quad B=\left\{\Sigma m_{\nu}, \mathrm{CP}\right\}$ where $\mathrm{CP}$ is a set of basic cosmological parameters: $\mathrm{CP}=$ $\left\{\Omega_{m}, \sigma_{8}, H_{0}, n_{s}\right\}$. We choose the best-fit model for WMAP7 as a fiducial model (Komatsu et al. [9]), namely, $\mathrm{CP}=\{0.266,0.801,71,0.93\}$, and choose $B_{0}=10^{-5}$ and $\Sigma m_{\nu}=0.05 \mathrm{eV}$ as fiducial values for models A and B respectively. We also marginalize over the nuisance parameter $C$ in Eq (2.3), whose fiducial value was evaluated using the $\Lambda \mathrm{CDM}$ model. We also combine the Fisher matrices for the current data of CMB (WMAP Komatsu et al. [9]) and SNe (UNION2 Amanullah et al. [1]) to improve the constraint (see details for CMB and SNe Fisher matrices in Pogosian et al. [12], Zhao et al. [21]). The result is shown in Fig 3. As we can see, the constraints on $B_{0}$ and neutrino mass are largely improved when the $\mathrm{CMN}$ data from $6 \mathrm{dF}$ is combined. Comparing with the constraints without CMN but only WMAP7 combined with UNION2 data, Giannantonio et al. [5] gives the 95\% CL. upper limits on $B_{0}$ as $B_{0}<0.4$ and Komatsu et al. [9] gives $\Sigma m_{\nu}<1.3 \mathrm{eV}$. The constraints can be tightened to $B_{0}<5 \times 10^{-5}$ and $\Sigma m_{\nu}<0.65 \mathrm{eV}$ when the $\mathrm{CMN}$ data from $6 \mathrm{dF}$ is included.

\section{Conclusion}

We provide a statistical tool to measure the $\mathrm{CMN}$, and further demonstrate that it is a sensitive probe of the structure growth. By design, the CMN is immune to the uncertainty in the overall amplitude of 
the density perturbation, and to linear galaxy bias. Also it is highly sensitive to any scale-dependent distortion of $P_{\theta \theta}$ since any small distortion can be amplified via the integral effect. Therefore it is an excellent tool to test alternative theories of gravity, and models with non-zero neutrino mass.

We first perform a likelihood analysis of the CMN from the current peculiar velocity field data, and further confront the WMAP7 $\Lambda$ CDM model and 1990s popular CDM model with the CMN data from current and old observations. We confirm that the $\Lambda$ CDM model with WMAP 7-yr parameters is consistent with current CMN data at $3 \sigma \mathrm{CL}$. level. Based on our forecast for $6 \mathrm{dF}$, we find that the $\mathrm{CMN}$ can improve the constraints on the modified gravity parameter $B_{0}$ by 4 orders of magnitude for the fiducial $B_{0}$ value of $10^{-5}$, and it can also tighten the present constraints on the neutrino mass. The CMN information from future surveys, such as the Square Kilometre Array (SKA) [22], will be more powerful to constrain cosmologies, especially for modified gravity models, and models with massive neutrinos.

\section{Acknowledgments}

We would like to thank A. Challinor, G. Efstathiou, K. Koyama and K. Masters for helpful discussions. GBZ is supported by STFC grant ST/H002774/1.

\section{References}

[1] Amanullah R. et al., 2010, ApJ, 716, 712

[2] Atrio-Barandela F., Kashlinsky A., Mucket J. P., 2004, ApJ, 601, 111

[3] Bernardi M. et al., 2002, Astron. J, 123, 2990

[4] da Costa L. N. et al., 2000, Astron. J, 120, 95

[5] Giannantonio T., Martinelli M., Silvestri A., Melchiorri A., 2010, JCAP, 04, 030

[6] Gramann M., Bahcall N. A., Cen R. and Gott J. R., 1995, ApJ, 441, 449

[7] Jennings E., Baugh C., Pascoli S., 2011, MNRAS 410, 2081

[8] Jones D.H. et al., 2009, MNRAS, 399, 683

[9] Komatsu E. et al., 2011, ApJS, 192, 18

[10] Nagamine K., Ostriker J. P., Cen R., 2001, ApJ, 553, 513

[11] Ostriker J. P., Suto Y., 1990, ApJ, 348, 378

[12] Pogosian L. et al., 2005, Phys. Rev. D., 72, 103519

[13] Springob C. M. et al., 2007, ApJS, 172, 599

[14] Strauss M. A., Cen R., Ostriker J. P., 1993, ApJ, 408, 389.

[15] Suto Y., Gouda N., Sugiyma N., 1990 ApJS, 74, 665

[16] Suto Y., Cen R., Ostriker J. P., 1992 ApJ, 395, 1

[17] Tegmark M., Taylor A., Heavens A., 1997, ApJ, 480, 22

[18] Tonry J. L. et al., 2001, ApJ, 546, 681

[19] Tonry J. L. et al., 2003, ApJ, 594, 1

[20] Wenger G. et al., 2003, Astron. J, 126, 2268

[21] Zhao G. B., Pogosian L., Silvestri A., Zylberberg J., 2009, Phys. Rev. D, 79, 083513

[22] http://www.skatelescope.org 